\title{
Endosperm starch grains of Andropogon, Arthraxon hispidus, and Hyparrhenia rufa (Andropogoneae, Panicoideae, Poaceae)
} Acta Botanica Mexicana

\author{
J. Gabriel Sánchez-Ken², [D
}

\begin{abstract}
:
Background and Aims: Grasses have five different types of endosperm starch grain morphology. Even though there is high diversity within the family, the morphology of the starch grains is generally represented by one or two species. Some genera, such as Andropogon (Andropogoneae), were reported to have at least three types of starch grains. However, most of the reviewed species were transferred to other genera. Therefore, the question whether the genus has one or more types of starch grain morphology remains unanswered.

Methods: Between four and eight mature caryopses were removed from specimens deposited in the herbarium IEB for most species, as well as from plants monitored in the field until they had mature caryopses. The caryopses were attached on a plate with a drop of white adhesive Resistol ${ }^{\circledR}$ or resin and then sectioned with a razor blade. Sections were stained with a drop of diluted Lugol's solution, microscopically observed and photographed at several magnifications.

Key results: All Andropogon species observed have only one type of starch grain morphology, the Andropogon-type. In all species the simple starch grains are much more abundant than the compound ones, except in $A$. tenuispatheus where the ratio is inverted. The other two reviewed species, Arthraxon hispidus and Hyparrhenia rufa have Andropogon-type and Panicum-type starch grains, respectively.

Conclusions: It is confirmed that, so far, all Andropogon species observed have one type (Andropogon-type) of endosperm starch grain morphology. There is variation in the size, size distribution and shape of the starch grains among the species. Andropogon gayanus is the only reviewed species with large starch grains reaching $28 \mu \mathrm{m}$, whereas those in the other species measure up to $15 \mu \mathrm{m}$ in diameter.
\end{abstract}

Key words: Andropogon fastigiatus, Andropogon virginicus complex, granules, hilum, Miscanthus-type, Triticum-type.

\section{Resumen:}

Antecedentes y Objetivos: Las gramíneas tienen cinco tipos diferentes de morfología de granos de almidón del endospermo; sin embargo, debido a la alta diversidad dentro de la familia, la morfología de los granos de almidón generalmente está representada por una o dos especies. Para géneros como Andropogon (Andropogoneae), se ha reportado que tiene al menos tres tipos de granos de endospermo; sin embargo, la mayoría de las especies que fueron revisadas han sido transferidas a otros géneros. Por lo tanto, la pregunta de si el género tiene uno o más tipos de morfología de los granos de almidón del endospermo, aún permanece sin contestar.

Métodos: Se removieron entre cuatro y ocho cariópsides maduras de especímenes depositados en el herbario IEB y de algunas especies que fueron monitoreadas en el campo hasta que tuvieran cariópsides maduras. Las cariópsides fueron fijadas en una placa con una gota de adhesivo blanco Resistol ${ }^{\circledR}$ o resina. Se hicieron los cortes con una navaja de rasurar muy delgada. Los cortes se tiñeron con una gota diluida de solución Lugol, se observaron al microscopio y se tomaron fotografías a diferentes amplificaciones.

Resultados clave: Todas las especies de Andropogon tienen solo un tipo de morfología de granos de almidón, el tipo-Andropogon. En todas las especies los granos de almidón simples son más abundantes que los compuestos, excepto $A$. tenuispatheus donde sucede lo contrario. Las otras dos especies revisadas, Arthraxon hispidus e Hyparrhenia rufa tienen tipo-Andropogon y tipo-Panicum, respectivamente.

Conclusiones: Hasta este momento, se confirma que todas las especies revisadas tienen un solo tipo de morfología (tipo-Andropogon) de granos de almidón del endospermo. Existe variación en el tamaño, distribución de tamaños y formas de granos de almidón entre las especies. Andropogon gayanus es la única especie que tiene granos de almidón grandes hasta $28 \mu \mathrm{m}$, mientras que en las otras especies estos pueden medir hasta $15 \mu \mathrm{m}$ de diámetro.

Palabras clave: Andropogon fastigiatus, complejo Andropogon virginicus, gránulos, hilio, tipo-Miscanthus, tipo-Triticum.

${ }^{1}$ Instituto de Ecología, A.C., Centro Regional del Bajío, Red de Diversidad Biológica del Occidente Mexicano, Av. Lázaro Cárdenas 253, Col. Centro, 61600 Pátzcuaro, Michoacán, Mexico.

${ }^{2}$ Author for correspondence: gabriel.sanchez@inecol. $\mathrm{mx}$
Received: July 8, 2021.

Reviewed: September 2, 2021.

Accepted by Marie-Stéphanie Samain: October 20, 2021

Published Online first: October 31, 2021

Published: Acta Botanica Mexicana 128 (2021).
To cite as: Sánchez-Ken, J. G. 2021. Endosperm starch grains of Andropogon, Arthraxon, and Hyparrhenia rufa (Andropogoneae, Panicoideae, Poaceae). Acta Botanica Mexicana 128: e1921. DOI: https://doi. org/10.21829/abm128.2021.1921


commercial Licence (CC BY-NC 4.0 Internacional). 


\section{Introduction}

Grass starch grains have been studied from the phylogenetic point of view, as well as because of their importance as a source of food from all edible grains and cereals (Sarwar et al., 2013). There are several classifications, some of them very detailed (Reichert, 1913) and others simplified, depending on the group (Tateoka, 1954, 1962; GPWG, 2001; Matsushima et al., 2013; Matsushima, 2015). The first classifications were inclusive for all plants with at least 17 different types of starch grains from approximately 137 genera and 346 species of grasses (Reichert, 1913). Tateoka (1954) observed 37 and 172 Pooideae genera and species, respectively, and Tateoka (1962), in a broader review, described four types of starch grains for 244 genera and 766 species throughout the family. Tateoka's (1962) classification includes whether the grains are simple or compound, with only one or two sizes and the number and size of the granules in the compound grains.

The current classification of starch grains in Poaceae is based on five types of morphologies, based on Tateoka (1962), and modified by the GPWG (2001). Type 1 or Triticum-type was designated for simple starch grains with shapes ranging from broadly elliptic, elliptic-round to rarely reniform, from 4-6 $\mu \mathrm{m}$ to medium-large size between 30$40 \mu \mathrm{m}$. This type of starch grain is common in the Pooideae subfamily. There are taxa with homogenous or unimodal sizes and heterogeneous or bimodal size starch grains. Type 2 or Panicum-type includes simple, round, polygonal to rarely rectangular starch grains, commonly 4-6 $\mu \mathrm{m}$, but some genera with large grains measuring between 30-40 $\mu \mathrm{m}$. This type of starch grains is common in Panicoideae, as well as in some taxa from other subfamilies such as Chloridoideae, Bambusoideae, Pooideae and Micrairoideae (Tateoka, 1962).

Type 3 (Miscanthus-type) or Andropogon-type is a mixture of simple and compound starch grains, where the first are round to polygonal (like Panicum-type) and between 15-40 $\mu \mathrm{m}$ in diameter. Commonly, compound grains consist of 2-4 granules, which are usually slightly smaller than simple grains. Depending on the ratio between simple and compound starch grains, the most common is where the simple starch grains are much more numerous, a second ratio is when both simple and compound starch grains are somewhat the same in some species, and a third ratio, perhaps the most uncommon, where the compound starch grains are numerous. This type of starch grain is common in Andropogoneae, some Chloridoideae and Arundinoideae taxa. Finally, Type 4 or Festuca-type is recognized in species where most of the grains are compound, each with few to many tiny granules between 2-6 $\mu \mathrm{m}$ in diameter (Judziewicz and Soderstrom, 1989; Bultosa et al., 2002; Yun and Kawagoe, 2010; Ai et al., 2016).

GPWG (2001) segregated the fifth type calling it Brachyelytrum-type, where all the starch grains are only large, between 30-40 $\mu \mathrm{m}$ diameter, without the smaller grains typical of Panicum-type. This difference had also already been noticed by Tateoka (1962).

Tateoka (1954) reviewed six species of andropogonoid genera, half of them with Panicum-type and the other half with compound grains. The author did not explain if the last three species had both simple and compound grains, but the illustration shows compound one with 2-4 and 13 granules, likely the Andropogon-type. Later, in a review of 49 species from 28 genera of the tribe Andropogoneae (unfortunately no names for the species were given), Tateoka (1962) found three types of grains. Fourteen genera have exclusively the most common Panicumtype, four Andropogon-type, six with Festuca-type, three with Panicum-type and Andropogon-type, and two with Panicum-type and Festuca-type. At the species level, 30 have Panicum-type, ten Festuca-type, and only eight species Andropogon-type (Table 1).

The starch grains of Andropogon L. are known only from two out of the 100-120 species recognized in the genus (Campbell, 2003; Nagahama and Norrmann, 2012). Previously, Reichert (1913) described the starch grains of 14 species assigned to Andropogon, from which only two were within the genus and the remaining 12 belonged to other genera. These two species, Andropogon leucostachyus Kunth and Andropogon virginicus L. have Andropogon-type starch grains (Reichert, 1913). Summarizing Reichert's (1913) and Tateoka's (1962) findings, it means that Andropogon has three types of starch grain morphology, Panicum-type, Andropogon-type, and Festuca-type, having in mind that Tateoka's (1962) studied species remain nameless. Perhaps this variation or uncertainty led Watson 
Table 1: List of genera and number of species of the tribe Andropogoneae with known starch grain types (taken from Tateoka (1962)). Boldface indicates genera with more than one type of starch grains.

\begin{tabular}{|c|c|c|c|}
\hline & & Species number & \\
\hline Genus & Panicum-type & Andropogon-type & Festuca-type \\
\hline *Andropogon L. & 7 & & 1 \\
\hline Apluda L. & & & 1 \\
\hline *Arthraxon P. Beauv. & 1 & 1 & \\
\hline Bothriochloa Kuntze & 1 & & \\
\hline${ }^{*}$ Chrysopogon Trin. & 1 & 1 & \\
\hline Cleistachne Benth. & 1 & & \\
\hline Coix L. & 2 & & \\
\hline Cymbopogon Spreng. & & 1 & \\
\hline Dichanthium Willimet & 1 & & \\
\hline Dimeria R. Br. & 1 & & \\
\hline Eremochloa Buse & 2 & & \\
\hline Erianthus Michx. & & & 1 \\
\hline Hemarthria R. Br. & 1 & & \\
\hline Heteropogon Pers. & 1 & & \\
\hline Hyparrhenia Andersson ex E. Fourn. & 1 & & \\
\hline *Ischaemum L. & 1 & & 3 \\
\hline Manisuris L. & & & 1 \\
\hline *Microstegium Nees & 1 & 1 & \\
\hline Miscanthus Andersson & & 2 & \\
\hline Mnesithea Hack. & & & 1 \\
\hline Phacelurus Griseb. & & & 1 \\
\hline Pogonatherum P. Beauv. & & & 1 \\
\hline Sorghastrum Nash & 1 & & \\
\hline Sorghum Moench & 2 & & \\
\hline Spodiopogon Trin. & 3 & & \\
\hline Themeda Forssk. & & 2 & \\
\hline Zea L. & 1 & & \\
\hline
\end{tabular}

et al. (1992) to leave out this information in the generic description of Andropogon. A different variation within a genus was observed by Matsushima et al. (2013): bimodal sizes of simple starch grains in Bromus catharticus Vahl. However, the genus typically has Triticum-type unimodal starch grains. Several other authors had reported only one size simple starch grains for the genus Bromus L. (Reichert, 1913; Tateoka, 1962; Musaubach et al., 2013).

Regarding Arthraxon P. Beauv. and Hyparrhenia Andersson ex E. Fourn., the former was reported to have two different types of starch grains (no species names given), Panicum-type and Andropogon-type (Tateoka, 1962) and the latter with Panicum-type grains (Reichert, 1913; Tateoka, 1962).

The objective of this research was to verify the variation of the starch grains among several species of Andropogon and to report the type of grains of Arthraxon hispidus (Thunb.) Makino and Hyparrhenia rufa (Nees) Stapf.

\section{Materials and Methods}

Grass specimens from the herbarium IEB (Centro Regional del Bajío, Instituto de Ecología, A.C.) were reviewed to see if they have developed caryopses, and those species 
that were nearby Pátzcuaro were monitored and collected when the caryopses were well developed. All collected vouchers were deposited in the herbarium IEB and duplicates will be sent out to the MEXU Herbarium (Instituto de Biología, Universidad Nacional Autónoma de México) and the herbarium EBUM (Universidad Michoacana de San Nicolás de Hidalgo) (acronyms according to Thiers, 2020). Four to eight caryopses were removed from each voucher. Caryopses of Arthraxon hispidus and Hyparrhenia rufa were also included to verify the morphology of their starch grains. Due to the tiny size of the caryopses, they were attached to a plate with a drop of white adhesive Resistol ${ }^{\circledR}$ or epoxy resin. Once the glue had dried, the caryopses were sectioned with a very thin razor blade. Starch grains were stained with $5 \%$ stock Lugol's solution (Potassium iodine) diluted 1:5 to 1:20 (Reichert, 1913; McNair, 1930). Stained sections were observed using a light microscope (Primo Star, Carl Zeiss, New York, USA), and an IPhone 11 (Apple, California, USA) to take photographs at several magnifications. The grains were measured using an eyepiece micrometer, calibrated with a stage micrometer. All measurements were made to include from the smallest to the largest grain; when possible, in the whole stained section, when the compound grains were scarce, all of them were measured. Photos were edited with GIMP v. 10.12.22 (Kimball et al., 1995-2021). Relative starch grain packing based on the proximity of the grains is characterized by the following traits: loosely packed where the grains are widely separated from each other, closely packed where the grains are close to each other, but not causing pressure, and tightly packed where the grains tightly touched each other, causing flattened facets due to pressure. Relative abundance is defined by the number of compound or simple grains: rarely present (1), low abundance (2-5), moderate abundance (6-30), and very high abundance (630).

The morphology of the starch grains is a constant character within a given species as its biosynthesis and review of several individuals has been probed (Matsushima et al., 2013; Tetlow and Emes, 2017). Therefore an individual can represent the whole species. Below is the list of the specimen vouchers from which caryopses were extracted:
Andropogon fastigiatus Sw.: MEXICO. Michoacán, municipio La Huacana, ca. $0.5 \mathrm{~km}$ NE of Los Ranchos at western base of cerro El Barril, $18^{\circ} 42^{\prime} 20^{\prime \prime} \mathrm{N}, 102^{\circ} 00^{\prime} 30^{\prime \prime} \mathrm{W}$, shady ravine in thornscrub, $300 \mathrm{~m}, 09 . \mathrm{XI.2002,} \mathrm{V.} \mathrm{W.} \mathrm{Steinmann}$ 2973 (IEB).

Andropogon gayanus Kunth: MEXICO. Michoacán, municipio Huiramba, camino Morelia - Pátzcuaro, cerca del restaurante Campestre Los Pinos, $19^{\circ} 32^{\prime} 15^{\prime \prime} \mathrm{N}, 101^{\circ} 32^{\prime} 00^{\prime \prime O}$, pastizal secundario, 2101 m, 17.XI.2020, J. G. Sánchez-Ken 1091 (IEB, MEXU).

Andropogon gerardi Vitman: MEXICO. Michoacán, municipio Tzintzuntzan, km 9 de la carretera Tzintzuntzan - Pátzcuaro, $19^{\circ} 36^{\prime} 43^{\prime \prime} \mathrm{N}, 101^{\circ} 34^{\prime} 36^{\prime \prime} \mathrm{O}$, pastizal secundario, 2121 m, 28.X.2020, J. G. Sánchez-Ken 1082 (IEB).

Andropogon liebmannii Hack. var. liebmannii: MEXICO. Veracruz, municipio Benigno Mendoza, Mecayapan, $18^{\circ} 18^{\prime} 55^{\prime \prime} \mathrm{N}, 94^{\circ} 46^{\prime} 35^{\prime \prime} \mathrm{O}$, ladera, $295 \mathrm{~m}, 11 . V I I .1994, \mathrm{G}$. Castillo C. et al. 12484 (IEB).

Andropogon pringlei Scribn. \& Merr.: MEXICO. Michoacán, municipio Maravatío, La Nopalera, $12 \mathrm{~km}$ al SE de Maravatío, sobre la carretera a Tlalpujahua, $19^{\circ} 49^{\prime} 50^{\prime \prime} \mathrm{N}$, $100^{\circ} 21^{\prime} 34^{\prime \prime O}$, vestigios de bosque tropical caducifolio, 2100 m, 10.X.1991, J. Rzedowski 50991 (IEB, MEXU).

Andropogon selloanus (Hack.) Hack.: MEXICO. Tabasco, municipio Huimanguillo, km 27 de la desviación de HuimanguiIlo a Francisco Ruedas, $17^{\circ} 47^{\prime} 26^{\prime \prime} \mathrm{N}, 93^{\circ} 26^{\prime} 14^{\prime \prime O}$, sabanas, 16 m, 15.VII.1988, M. A. Magaña et al. 2056 (IEB).

Andropogon tenuispatheus (Nash) Nash: MEXICO. Michoacán, municipio Morelia, antes de llegar a Morelia, carrete-

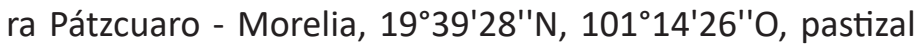
secundario en zona inundable, $1898 \mathrm{~m}, 10 . X I .2020$, J. G. Sánchez-Ken et al. 1089 (IEB, MEXU).

Andropogon virginicus L. var. virginicus: MEXICO. Veracruz, municipio Xalapa, carretera Xalapa - Coatepec, $19^{\circ} 27^{\prime} \mathrm{N}$, $98^{\circ} 56^{\prime} O$, bosque caducifolio con Liquidambar y encinar, 1150 m, 7.X.1980, T. Mejía Saulés 359 (IEB, XAL). 
Arthraxon hispidus (Thunb.) Makino: MEXICO. Michoacán, municipio Ziracuaretiro, Malpaís de San Andrés Corú, $19^{\circ} 27^{\prime} 31^{\prime \prime} \mathrm{N}, 101^{\circ} 56^{\prime} 47^{\prime \prime} \mathrm{O}$, bosque de pino-encino, $1685 \mathrm{~m}$, 12.XI.2012, D. Valentín M. 460 (IEB).

Hyparrhenia rufa (Nees) Stapf: MEXICO. Michoacán, municipio Morelia, carretera Pátzcuaro - Morelia, a $1.8 \mathrm{~km}$ al SW de La Estancia a un lado de la carretera, $19^{\circ} 34^{\prime} 34^{\prime \prime} \mathrm{N}$, $101^{\circ} 18^{\prime} 50^{\prime \prime O}$, pastizal secundario, 2018 m, 21.X.2020, J. G. Sánchez-Ken 1077 (IEB).

\section{Results}

Descriptions of the caryopses and starch grains of eight Andropogon species, Arthraxon hispidus and Hyparrhenia rufa are summarized in Table 2. The following list provides a detailed description of the caryopses and starch grains for each species.

Andropogon fastigiatus (Andropogon-type): caryopses fusiform, 3-3.4 × 1-1.2 mm wide, embryo 2-2.3 $\mathrm{mm}$ long, hilum punctiform, slightly triangular, 0.5-0.6 $\mathrm{mm}$ long (Fig. 1A); simple starch grains irregularly elliptic, triangular to somewhat polygonal with flat facets due to pressure (Fig. 2A), 5-11(-13) $\mu \mathrm{m}$ diameter, size distribution even to uneven towards the aleurone layer, tightly packed; compound starch grains 11-15 $\mu \mathrm{m}$ diameter, with (2-)3(-5) granules, moderate abundance; granules 5-8 $\mu \mathrm{m}$ diameter, circular to squarish, facets flat towards the center, rounded externally (Fig. 3A).

Andropogon gayanus (Andropogon-type): caryopses obovate to ovate, 3.4-3.5 × 1.2-1.3 mm wide, embryo 2-2.1 $\mathrm{mm}$ long, hilum punctiform, circular, 0.4-0.5 mm long (Fig. 1B); simple starch grains circular, irregularly circular, squarish to polygonal with flat facets due to pressure (Fig. 2B), (5-)825(-28) $\mu \mathrm{m}$ diameter, size distribution uneven towards the aleurone layer, closely packed; compound starch grains 7-18 $\mu \mathrm{m}$ diameter, with 3 granules, low abundance; granules (317-10 $\mu \mathrm{m}$ diameter, circular to slightly angular at the union, facets flat towards the union, rounded externally (Fig. 3B).

Table 2: Summary of the characters of the starch grains among the species observed in this study. Types of starch grains: $2=$ Panicum-type, 3=Andropogon-type, "3"=inverted Andropogon-type.

\begin{tabular}{|c|c|c|c|c|c|c|c|}
\hline Species & Type & $\begin{array}{l}\text { Simple grains } \\
\text { diameter }(\mu \mathrm{m})\end{array}$ & $\begin{array}{l}\text { Grains } \\
\text { packing }\end{array}$ & $\begin{array}{c}\text { Compound } \\
\text { grains } \\
\text { diameter }(\mu \mathrm{m})\end{array}$ & Abundancy & $\begin{array}{r}\text { Granules } \\
\text { number }\end{array}$ & $\begin{array}{c}\text { Granules } \\
\text { diameter } \\
(\mu \mathrm{m})\end{array}$ \\
\hline Andropogon fastigiatus Sw. & 3 & $5-11(-13)$ & tightly & $11-15$ & moderate & $(2-) 3(-5)$ & $5-8$ \\
\hline Andropogon gayanus Kunth & 3 & $(5-) 8-25(-28)$ & closely & $6-15$ & low & 3 & $(3-) 7-10$ \\
\hline Andropogon gerardi Vitman & 3 & $5-13(-14)$ & loosely & $10-13$ & moderate & $2-5(-6)$ & $4-6$ \\
\hline $\begin{array}{l}\text { Andropogon liebmannii Hack. } \\
\text { var. liebmannii }\end{array}$ & 3 & $(2-) 4-10(-12)$ & loosely & $8-13$ & low & $2-4$ & $4-6$ \\
\hline $\begin{array}{l}\text { Andropogon pringlei Scribn. \& } \\
\text { Merr. }\end{array}$ & 3 & $5-13(-15)$ & closely & $13-14$ & moderate & $2-4$ & $4-6$ \\
\hline $\begin{array}{l}\text { Andropogon selloanus (Hack.) } \\
\text { Hack. }\end{array}$ & 3 & $(3-) 5-11$ & loosely & $7-12$ & low & $2-3$ & $5-6$ \\
\hline $\begin{array}{l}\text { Andropogon tenuispatheus } \\
\text { (Nash) Nash }\end{array}$ & “3” & $3-10$ & closely & $(6-) 7-15$ & very high & $(2-) 3-9$ & $4-6$ \\
\hline $\begin{array}{l}\text { Andropogon virginicus L. var. } \\
\text { virginicus }\end{array}$ & 3 & $4-12(-14)$ & loosely & $10-13$ & low & $2-3$ & $3-7$ \\
\hline $\begin{array}{l}\text { Arthraxon hispidus (Thunb.) } \\
\text { Makino }\end{array}$ & 3 & $(3-) 5-10(-12)$ & loosely & $9-13$ & low & 3 & $4-9$ \\
\hline Hyparrhenia rufa Stapf & 2 & $9-18(-20)$ & tightly & - & - & - & - \\
\hline
\end{tabular}



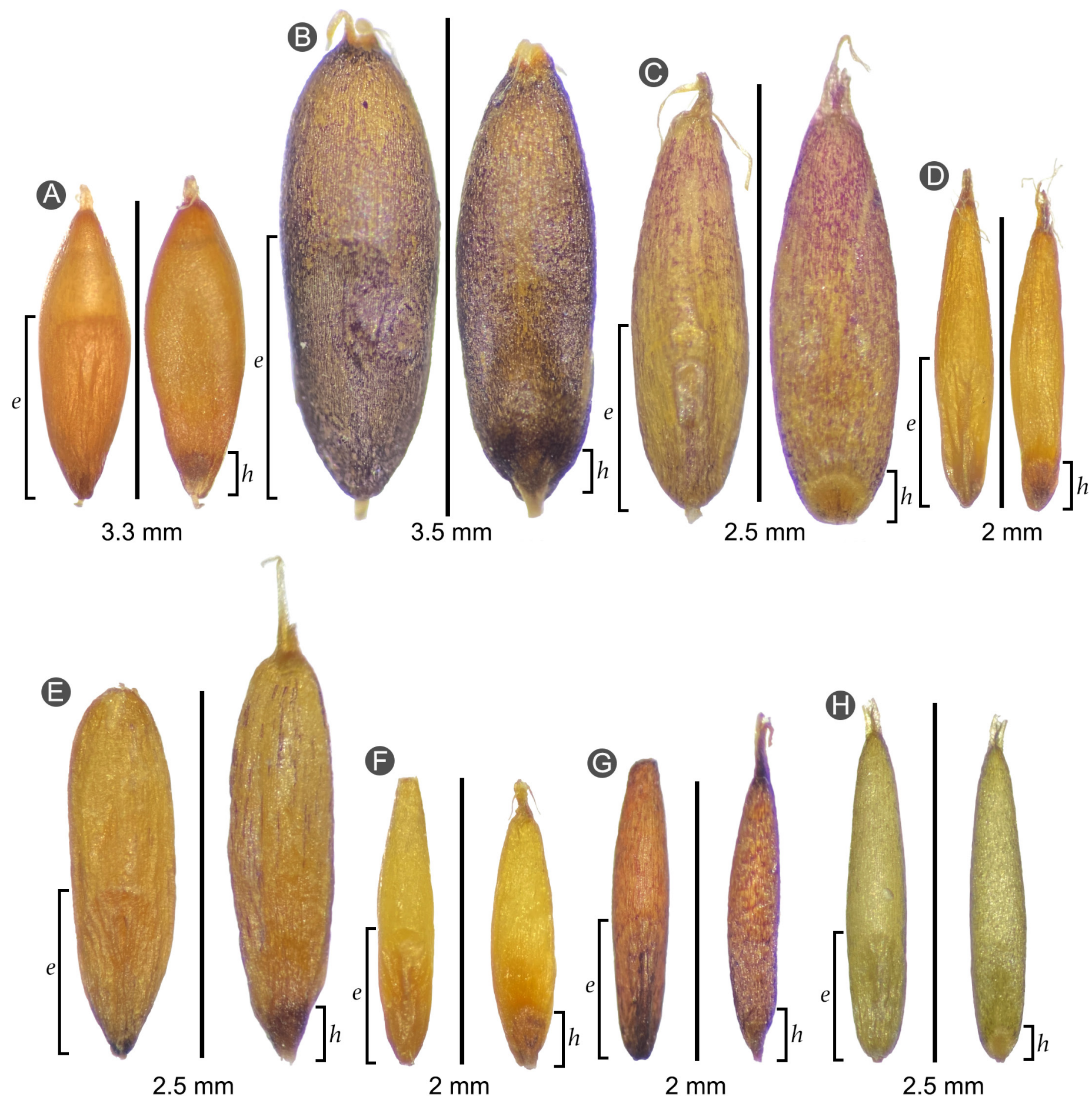

Figure 1: Ventral and dorsal views of Andropogon L. Caryopses: A. Andropogon fastigiatus Sw.; B. Andropogon gayanus Kunth; C. Andropogon gerardi Vitman; D. Andropogon liebmannii Hack. var. liebmannii; E. Andropogon pringlei Scribn. \& Merr.; F. Andropogon selloanus (Hack.) Hack.; G. Andropogon tenuispatheus (Nash) Nash.; H. Andropogon virginicus L. var. virginicus. e=embryo; $h=h i l u m$.

Andropogon gerardi (Andropogon-type): caryopses ovate, 2.4-2.5 × 0.6-0.7 mm wide, embryo 1.1-1.3 mm long, hilum punctiform, circular, 0.3-0.4 mm long (Fig. 1C); simple starch grains widely elliptic, irregularly elliptic, irregu- larly squarish to polygonal, some with flat facets (Fig. 2C), 5-13(-14) $\mu \mathrm{m}$ diameter, size distribution uneven, loosely packed; compound starch grains 10-13 $\mu \mathrm{m}$ diameter, with 2-5 granules, moderate abundance; granules 4-6 $\mu \mathrm{m}$, cir- 




Figure 2: Outlines of starch grains observed. A. Andropogon fastigiatus Sw.; B. Andropogon gayanus Kunth; C. Andropogon gerardi Vitman; D. Andropogon liebmannii Hack. var. liebmannii; E. Andropogon pringlei Scribn. \& Merr.; F. Andropogon selloanus (Hack.) Hack.; G. Andropogon tenuispatheus (Nash) Nash.; H. Andropogon virginicus L. var. virginicus; I. Arthraxon hispidus (Thunb.) Makino; J. Hyparrhenia rufa (Nees) Stapf.

cular to squarish, flat facets towards the center, rounded externally (Fig. 3C).

Andropogon liebmannii var. liebmannii (Andropogontype): caryopses ovate-elliptic, 1.9-2.1 × 0.4-0.5 mm wide, embryo 1-1.1 mm long, hilum punctiform, circular, 0.3-0.4 mm long (Fig. 1D); simple starch grains irregularly circular, widely elliptic, few triangular to squarish, with flat facets (Fig. 2D), (2-)4-10(-12) $\mu \mathrm{m}$ diameter, size distribution uneven, but larger towards the center, loosely packed; compound starch grains 8-13 $\mu \mathrm{m}$ diameter, with 2-4 granules, low abundance; granules 5-7 $\mu \mathrm{m}$ diameter, circular to polygonal, flat facets towards the center, rounded externally (Fig. 4A).
Andropogon pringlei (Andropogon-type): caryopses obovate, 2.5-2.6 × 0-4-0.5 mm wide, embryo 1.1-1.2 mm long, hilum punctiform, circular, 0.4-0.5 mm long (Fig. 1E); simple starch grains circular, irregularly circular, squarish to polygonal, some with flat facets (Fig. 2E), 5-13(-15) $\mu \mathrm{m}$ diameter, size distribution uneven, closely packed; compound starch grains 13-14 $\mu \mathrm{m}$ diameter, with 2-4 granules, moderate abundance; granules 4-6 $\mu \mathrm{m}$ diameter, circular to squarish, flat facets towards the center, rounded externally (Fig. 4B).

Andropogon selloanus (Andropogon-type): caryopses elliptic, 2-2.1 × 0.3-0.4 mm wide, embryo 0.9-1 mm long, hilum punctiform, triangular, 0.4-0.5 mm long (Fig 1F); simple starch grains circular, irregularly circular, squarish, some with somewhat flat facets (Fig. 2F), (3-)5-11 $\mu$ m diameter, size distribution uneven, loosely packed; compound starch grains 7-12 $\mu \mathrm{m}$, with 2-3 granules, low abundance; granules 5-6 $\mu \mathrm{m}$ diameter, circular to squarish, flat facets towards the center, rounded externally (Fig. 4C).

Andropogon tenuispatheus (previously A. glomeratus Britton, Sterns \& Poggenb. var. pumilus (Vasey) L.H. Dewey (Wipff and Shaw, 2018, "Andropogon-type"): caryopses elliptic, 2-2.1 $\times$ 0.4-0.5 mm wide, embryo 1-1.1 mm long, hilum punctiform, triangular, 0.3-0.4 mm long (Fig. 1G); simple starch grains circular to elliptic (Fig. 2G), 3-10 $\mu \mathrm{m}$ diameter, low abundance, sparsely distributed, closely packed; compound starch grains (6-)7-15 $\mu \mathrm{m}$ diameter, irregular, with (2-)3-9 granules, circular, squarish, triangular, larger compound grains towards the center, size distribution uneven, very high abundance; granules 4-6 $\mu \mathrm{m}$ diameter, circular to squarish, flat facets towards the center, rounded externally (Fig. 5A).

Andropogon virginicus var. virginicus (Andropogontype): caryopses ovate-elliptic, 2.4-2.5 $\times$ 0.6-0.7 mm wide, embryo 0.9-1 mm long, hilum punctiform, circular to slightly oblong, 0.2-0.3 mm long (Fig. 1H); simple starch grains circular to irregularly circular, irregularly elliptic (Fig. $2 \mathrm{H}$ ), 4-12(-14) $\mu \mathrm{m}$ diameter, size distribution uneven, larger towards the center, loosely packed; compound starch grains 10-13 $\mu \mathrm{m}$ diameter, with 2-3 granules, low abundance; 

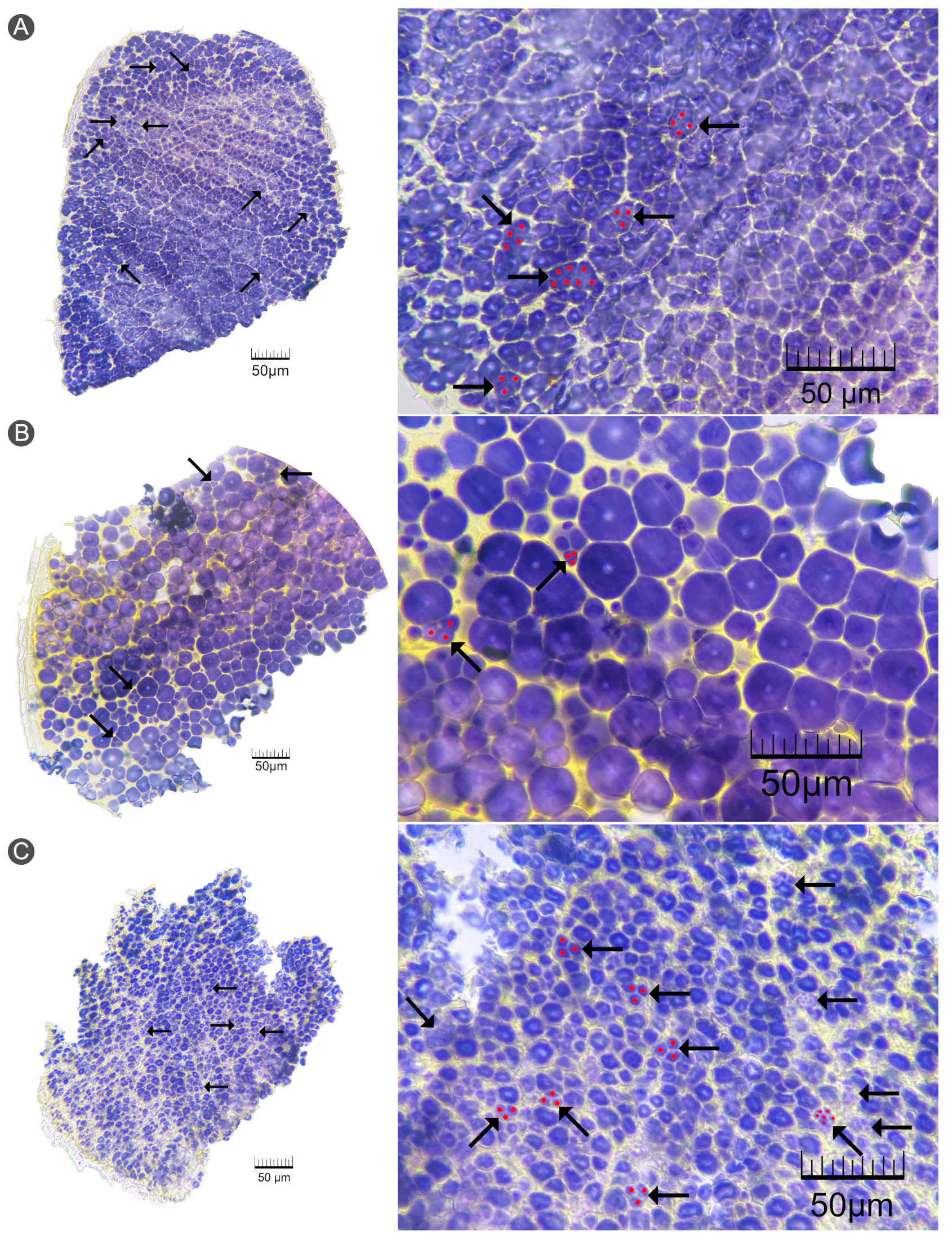

Figure 3: Starch grains. A. Andropogon fastigiatus Sw.; B. Andropogon gayanus Kunth; C. Andropgon gerardi Vitman. Left column=lower magnification; right column=higher magnification. Arrows point to compound starch grains; red dots are granules. 


\section{A}

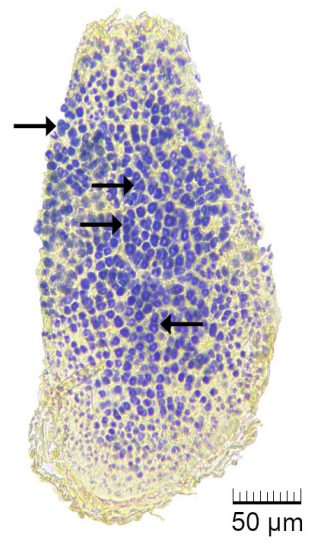

B

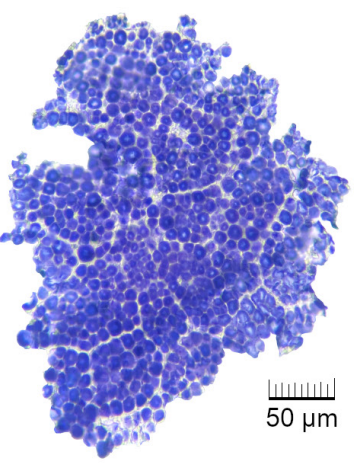

C

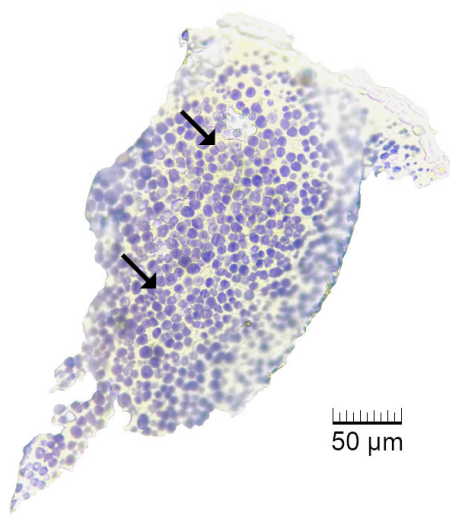

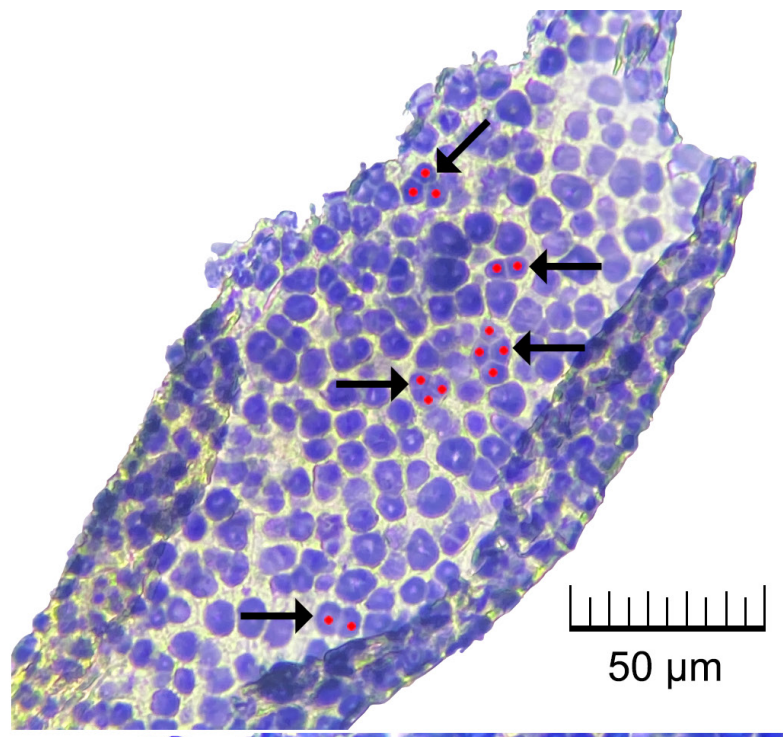
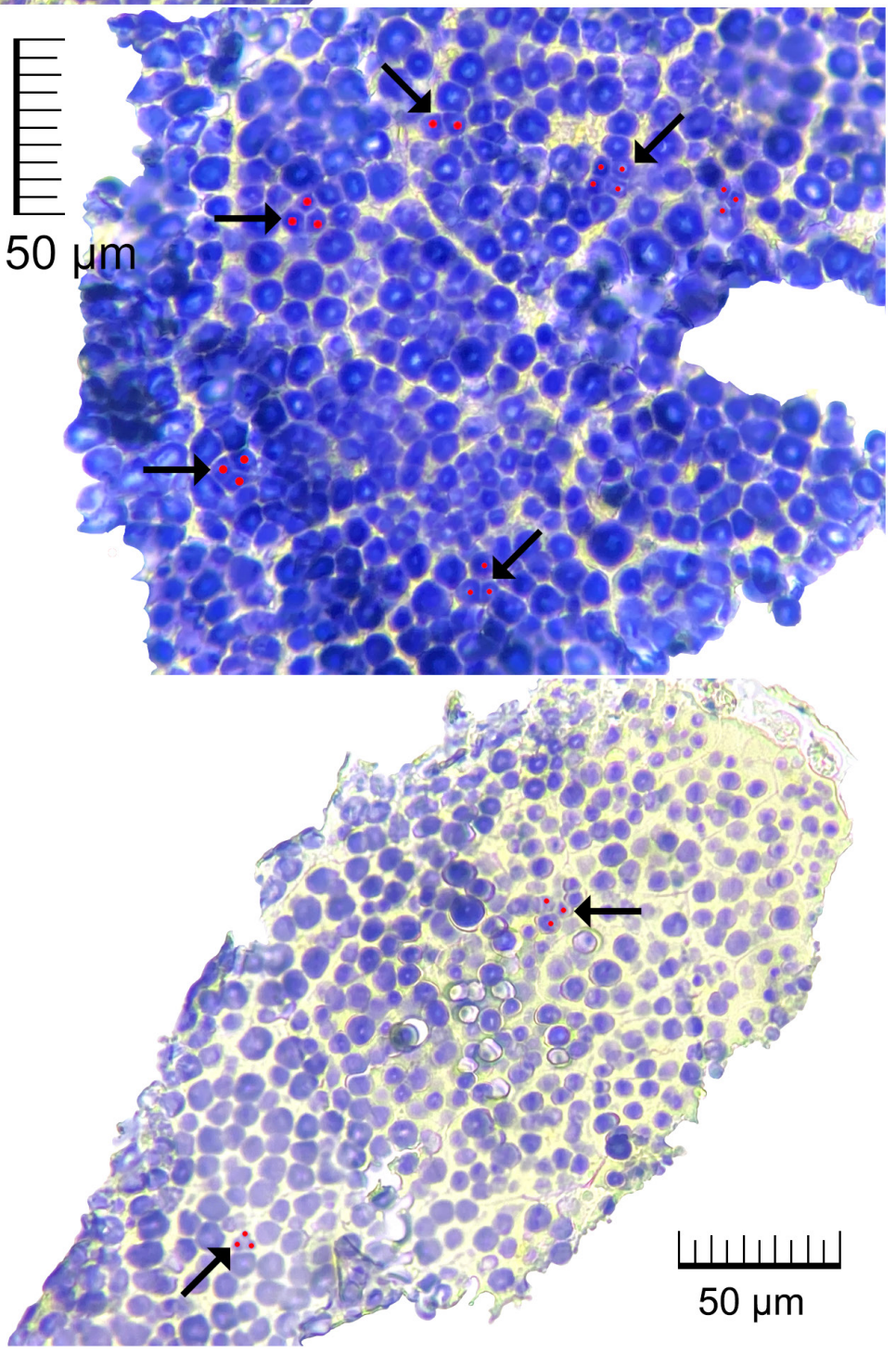

Figure 4: Starch grains. A. Andropogon liebmannii Hack. var. liebmannii; B. Andropogon pringlei Scribn. \& Merr.; C. Andropgon selloanus (Hack.) Hack. Left column=lower magnification; right column=higher magnification. Arrows point to compound starch grains; red dots are granules. 

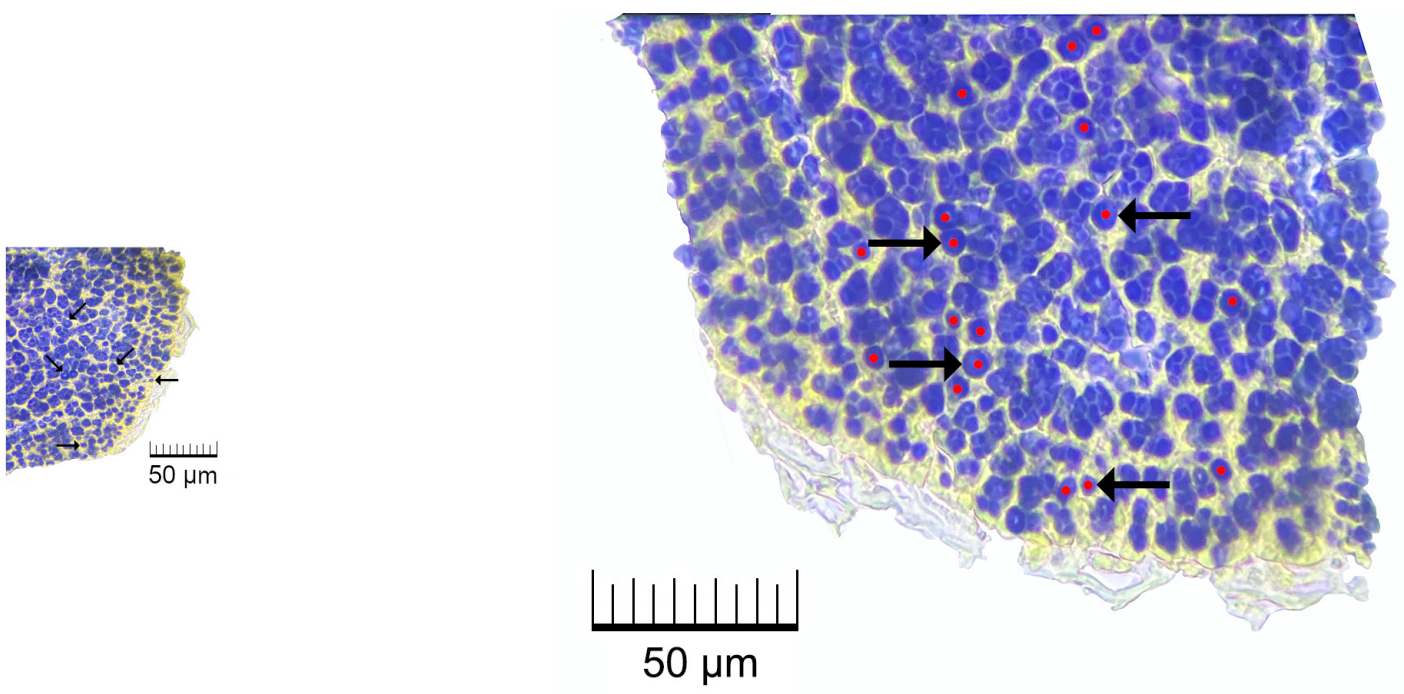

B
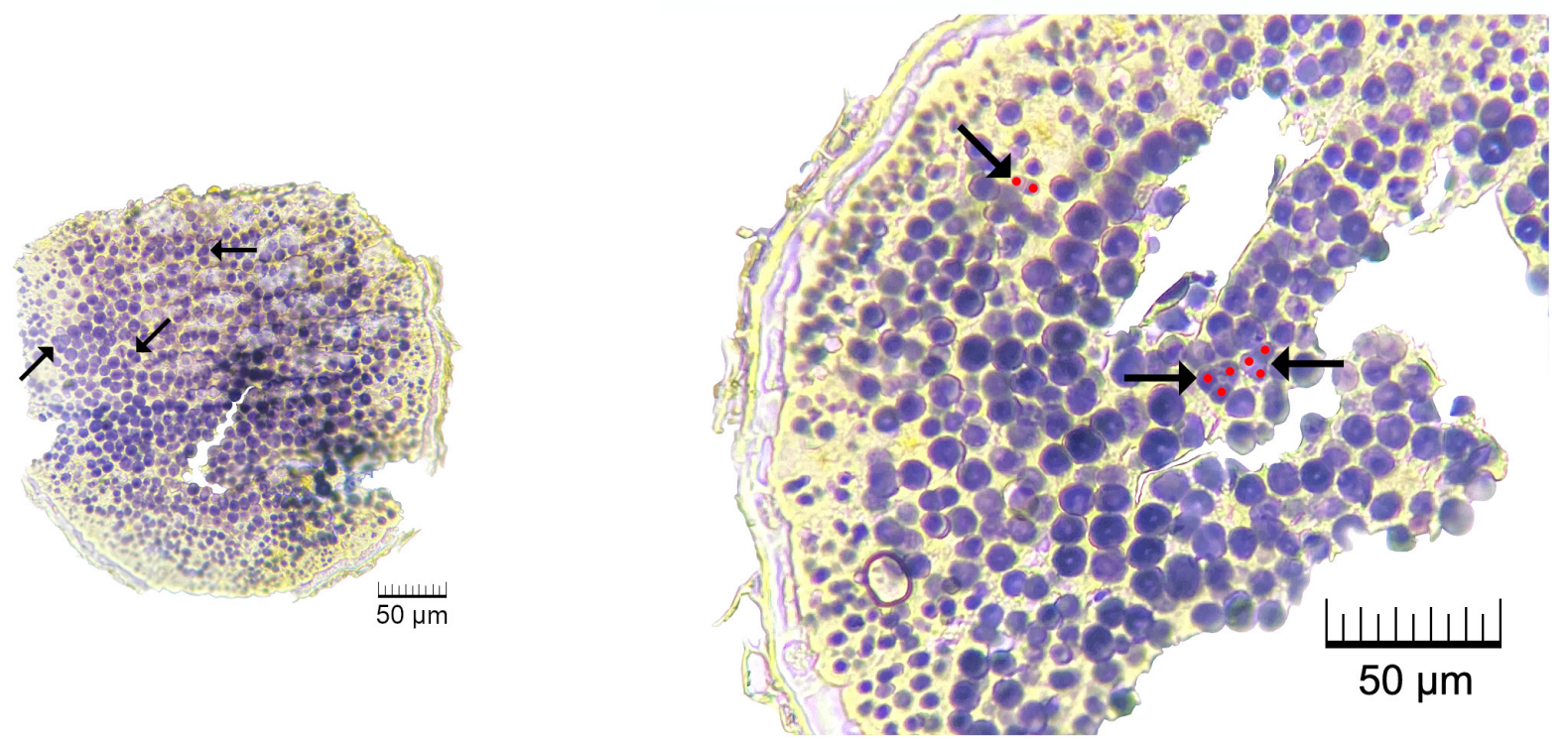

Figure 5: Starch grains. A. Andropogon tenuispatheus (Nash) Nash (arrows point to simple grains, red dots are simple grains); B. Andropogon virginicus L. var. virginicus (arrows point to compound starch grains, red dots are granules). Left column=lower magnification; right column=higher magnification.

granules 3-7 $\mu \mathrm{m}$ diameter, circular to squarish, flat facets towards the center, rounded externally (Fig. 5B).

Arthraxon hispidus (Andropogon-type): caryopses elliptic, $1.9-2 \times 0.2-0.3 \mathrm{~mm}$ wide, embryo 0.6-0.7 mm long, hilum punctiform, slightly oblong, 0.2-0.3 mm long (Fig. $6 \mathrm{~A})$; simple and compound starch grains; simple starch grains circular, irregularly elliptic to polygonal, some with flat facets due to pressure (Fig. 2I), (3-)5-10(-12) $\mu \mathrm{m}$ diameter, size distribution uneven, larger towards the center, closely packed; compound starch grains 9-13 $\mu \mathrm{m}$ diameter, with 3 granules, low abundance; granules 4-9 $\mu$ m diameter, circular to squarish, flat facets towards the center, rounded externally (Figs. 6A-C).

Hyparrhenia rufa (Panicum-type): caryopses obovate, 2.5-2.6 0 0.7-0.8 mm, embryo 1.1-1.2 mm long, hilum punctiform, triangular, 0.2-0.3 mm long (Fig. 6D); simple starch grains; simple grains triangular to polygonal with flat facets due to pressure, those towards the aleurone layer irregularly circular (Fig. 2J), 9-18(-20) $\mu \mathrm{m}$ diameter, larger grains towards the center, tightly packed (Figs. 6D-F). 
A



B

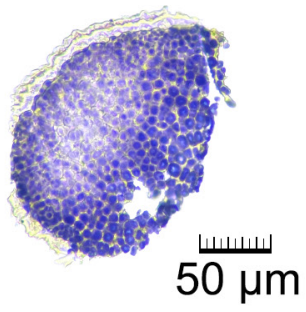

D

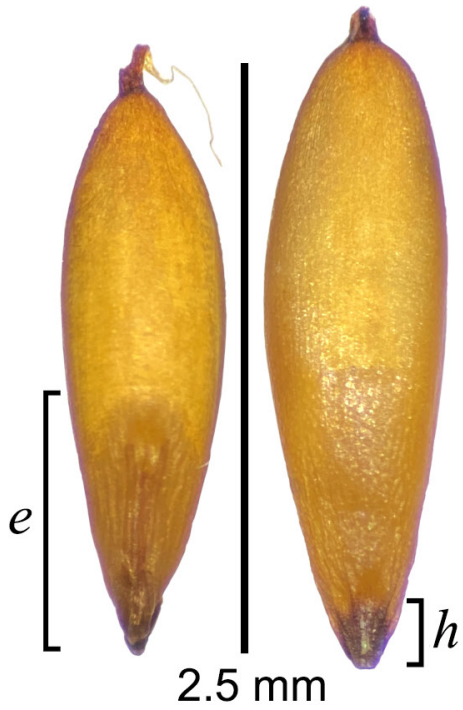

E
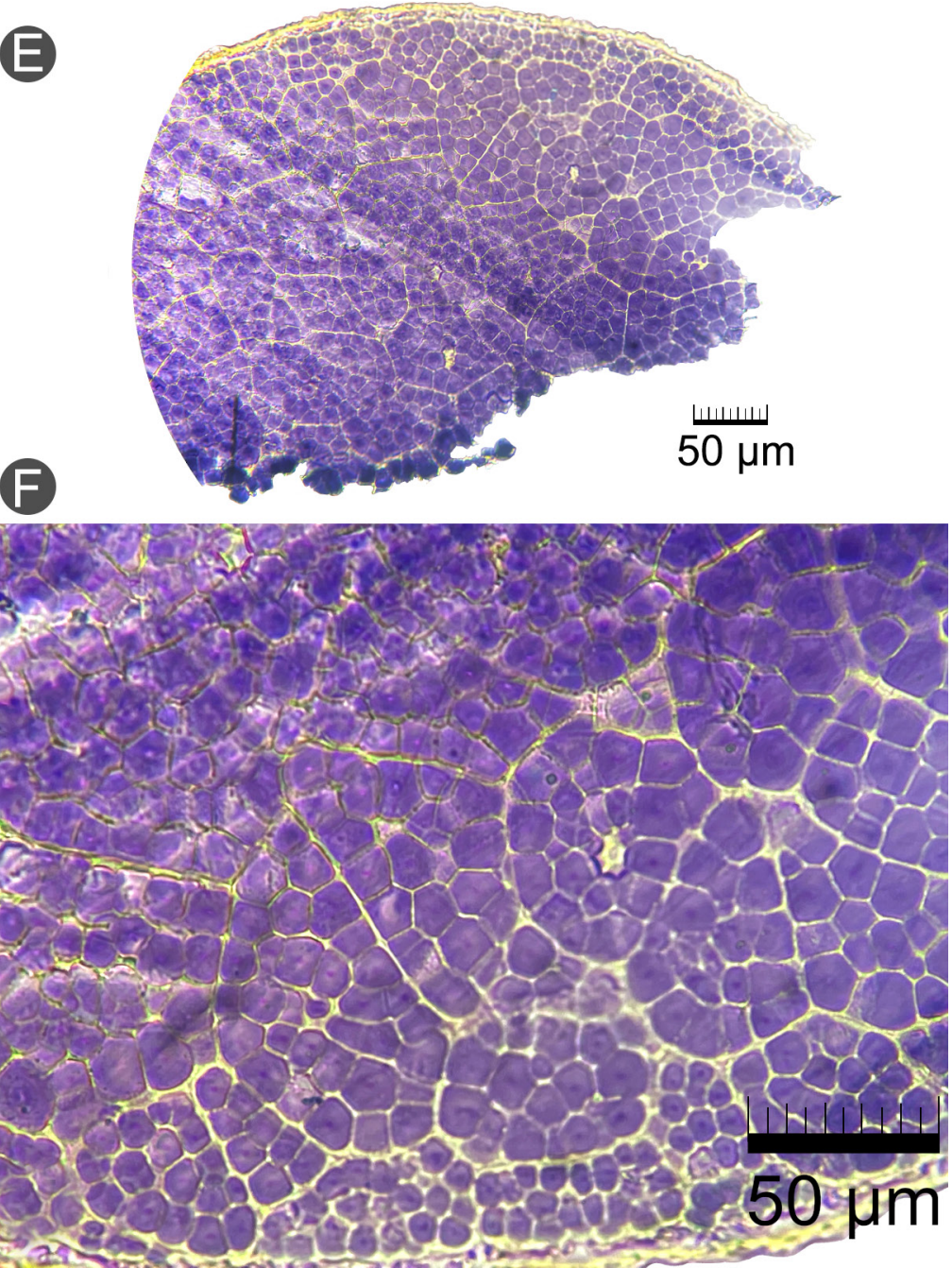

Figure 6: Caryopses and endosperm starch grains. A-C. Arthraxon hispidus (Thunb.) Makino: A. caryopses; B. endosperm in transversal view, lower magnification; C. endosperm in transversal view, higher magnification. D-F. Hyparrhenia rufa (Nees) Stapf: D. caryopses; E. endosperm in transversal view, lower magnification; F. endosperm intransversal view, higher magnification. Caryopses: Left=ventral view; right=dorsal view; $e=e m b r y o$; $h=$ hilum. 


\section{Discussion}

It has been assumed that the morphology of the starch grains within a genus is unique, except for some genera of the subfamilies Panicoideae and Bambusoideae (Tateoka, 1962 and references therein). Tateoka (1962) reviewed 766 species from 244 genera, from all these genera representing the whole family, most have only one type of starch grains. Few genera of some subtribes of Andropogoneae (Andropogon L., Arthraxon, Chrysopogon Trin., Ischaemum L., and Mischanthus Andersson), one genus from Paspaleae (Arthropogon Nees), and two genera of Paniceae (Panicum L. and Digitaria Haller), according to the current classification (Soreng et al., 2015), apparently have more than one type of starch grains. Several genera have been segregated from Andropogon and Panicum since then and because Tateoka (1962) only reported the number of the species but not their names, those species with a different type of starch grains remain unknown.

All the species of Andropogon and Arthraxon are characterized by Andropogon-type starch grains. Seven out of eight species have a major percentage of simple starch grains with low (2-5) to moderate abundance (630) of compound starch grains. These compound starch grains have two to five starch granules, except those in Andropogon tenuispatheus with up to nine granules. Among all these species, $A$. tenuispatheus has a unique morphology, an inverted Andropogon-type, where the compound starch grains are the most abundant compared to the simple grains, which may account for less than $5 \%$ of those observed. So far, this inverted Andropogon-type has been observed in Tridens chapmannii (Small) Chase, Chloridoideae), Thuarea sarmentosa Pers., Paniceae), and the Andropogoneae taxa Microstegium vimineum (Trin.) A. Camus, Chrysopogon aciculatus (Retz.) Trin., and Themeda triandra Forssk. (Tateoka, 1962).

Regarding the simple starch grains size, all Andropogon species range from (2-)3-13(-15) $\mu \mathrm{m}$ diameter (numbers in parenthesis indicate rare or unique grains with those sizes), whereas in $A$. gayanus the grains can reach up to $28 \mu \mathrm{m}$ diameter, more than twice the size of the grains of the other species. The arrangement of the grains may be tightly packed like in $A$. fastigiatus where the facets of the grains appear to be flattened; the same goes for those species with closely but not tightly packed in species such as $A$. gayanus and $A$. pringlei judging by the roundness of the grains. In the remaining species ( $A$. gerardi, $A$. liebmannii var. liebmannii, $A$. selloanus, and $A$. virginicus var. virginicus), the simple grains are loose, so the facets are rounded. In $A$. tenuispatheus the compound starch grains are closely packed but the facets of the granules are flattened because they are part of a compound grain.

Whether Andropogon fastigiatus belongs or not in the genus Andropogon, remains unclear (McAllister et al., 2018; Welker et al., 2020) but for the time being, it is still considered within the genus. The morphology of the caryopses and starch grains, at least in the reviewed species, is a little different, which may suggest that the species should be considered in its own genus, Diectomis Kunth. Tateoka (1962) reported Panicum-type starch grains for a nameless species, which does not agree with the Andropogon-type found here. Even though A. gerardi (sect. Andropogon L.) and $A$. pringlei (probably sect. Leptopogon Stapf) belong to different sections, both have similar caryopses and starch grains. Only the packing of the starch grains is different, in the former they are loosely packed and, in the latter, they are moderately packed. Regarding the caryopsis shape, all the remaining species ( $A$. liebmannii var. liebmannii, A. selloanus, $A$. tenuispatheus, and $A$. virginicus var. virginicus) belong to the section Leptopogon (Nagahama and Norrmann, 2012), sharing similar caryopses and morphology of the starch grains. From these four species, Reichert (1913) reported $A$. virginicus var. virginicus to have Andropogon-type starch grains, like those found here. Of these four species, three belong to the $A$. virginicus complex (A. liebmannii, A. tenuispatheus, and $A$. virginicus var. virginicus) according to Campbell (1983); A. liebmannii var. liebmannii and $A$. selloanus, a species putatively related to that complex appeared in a well-supported group of the section Leptopogon in the phylogenetic analysis by Welker et al. (2020). This assumption is also supported by the size and shape of the caryopses, as well as embryo and hilum length; however, they differ in the color of the pericarp and the shape of the hila. Starch grains are similar in A. liebmanni var. liebmanni, A. selloanus, and A. virginicus var. virginicus, in which the simple starch grains are more common than the compound ones. The opposite occurs in 
A. tenuispatheus, where the compound starch grains are the most common compared to the simple ones, a condition also found in Gymnopogon spicatus (Spreng.) Kuntze, Digitaria ciliaris (Retz.) Koeler (previously D. adscendens (Kunth) Henrard) and other taxa mentioned by Tateoka (1962).

Tateoka (1962) only concluded that Arthraxon had Panicum-type and Andropogon-type without naming the species. Arthraxon hispidus is very similar to the species of Andropogon reviewed here having the Andropogontype.

Finally, Hyparrhenia is in the same situation of Andropogon; its monophyly is not clear yet. However, the Panicum-type description given by Reichert (1913) is like in this study: the simple grains of $H$. rufa are large, circular with flattened facets due to pressure, in the range size of 9-18(-20) $\mu \mathrm{m}$ diameter, a range a little smaller than the 15-25 $\mu \mathrm{m}$ diameter reported by Reichert (1913) for several species of Hyparrhenia.

\section{Conclusions}

It is confirmed so far that all the reviewed Andropogon species have only one type of endosperm starch grain morphology, the Andropogon-type. However, because the species names studied earlier remain nameless, the Panicumtype and Festuca-type are still considered for the genus. Andropogon fastigiatus or Diectomis fastigiata (Sw.) P. Beauv. and Arthraxon hispidus have Andropogon-type and Hyparrhenia rufa has Panicum-type starch grains.

\section{Author contributions}

JGSK conceived and designed the study, reviewed the collections, realized the anatomical study, edited the photographs, wrote, and edited the manuscript after the revision.

\section{Funding}

This study was funded by the operative budget of the author from the Instituto de Ecología, A.C. (INECOL).

\section{Acknowledgements}

I would like to acknowledge Brenda Y. Bedolla García and Carlos A. Cultid Medina from INECOL for their support in the field.

\section{Literature cited}

Ai, Y., L. Gong, M. Reed, J. Huag, Y. Zhang and J. L. Jane. 2016. Characterization of starch from bamboo seeds. Starch 68(12): 131-139. DOI: https://doi.org/10.1002/star.201500206

Bultosa, G., A. N. Hall and J. R. N. Taylor. 2002. Physico-chemical characterization of grain Tef (Eragrostis tef (Zucc.) Trotter) starch. Starch 54(10): 461-468. DOI: https:// doi.org/10.1002/1521-379x(200210)54:10<461::AIDSTAR461>3.0.CO;2-U

Campbell, C. S. 1983. Systematics of the Andropogon virginicus complex (Gramineae). Journal of the Arnold Arboretum 64(2): 171-254. DOI: https://doi.org/10.5962/bhl. part.27406

Campbell, C. S. 2003. Andropogon L. In: Barkworth, M. E., K. M. Capels, S. Long and M. B. Piep (eds.). Flora of North America, North of Mexico. Magnoliophyta: Commelinidae (in part): Poaceae, part 2. Oxford University Press. New York, USA. Pp. 649-664.

GPWG. 2001. Grass Phylogeny Working Group. Phylogeny and subfamilial classification of the grasses (Poaceae). Annals of the Missouri Botanical Garden 88(3): 373-457. DOI: https:// doi.org/10.2307/3298585

Judziewicz, E. J. and T. R. Soderstrom. 1989. Morphological, anatomical, and taxonomic studies in Anomochloa and Streptochaeta (Poaceae: Bambusoideae). Smithsonian Contributions to Botany 68: 1-52. DOI: https://doi. org/10.5962/bhl.title.131652

Kimball, S., P. Mattis and the GIMP Development Team. 19952021. GNU Image Manipulation Program @ GIMP 2.10.22. Creative Commons Attribution-ShareAlike 4.0 International License.

Matsushima, R. 2015. Morphological variations of starch grains. In: Nakamura, Y. (ed.). Starch, metabolism and structure. Springer. Tokyo, Japan. 425-442 pp. DOI: https://doi. org/10.1007/978-4-431-55495-0_13

Matsushima, R., J. Yamashita, S. Kariyama, T. Enomoto and W. Sakamoto. 2013. A phylogenetic re-evaluation of morphological variations of starch grains among Poaceae species. Journal of Applied Glycoscience 60: 37-44. DOI: https://doi.org/10.5458/jag.jag.JAG-2012_006

McAllister, C. A., M. R. McKain, M. Li, B. Bookout and E. A. Kellogg. 2018. Specimen-based analysis of morphology and the environment in ecologically dominant grasses: the power of 
the herbarium. Philosophical Transactions B 374: 20170403. DOI: https://doi.org/10.1098/rstb.2017.0403

McNair, J. B. 1930. The differential analysis of starches. Field Museum of Natural History, Botany 9(1): 1-44. DOI: https:// doi.org/10.5962/bhl.title.2312

Musaubach, M. G., A. Plos and M. P. Babot. 2013. Differentiation of archaeological maize (Zea mays L.) from native wild grasses based on starch grain morphology. Cases from the Central Pampas of Argentine. Journal of Archaeological Science 40(2): 1186-1193. DOI: https://doi.org/10.1016/j. jas.2012.09.026

Nagahama, N. and G. A. Norrmann. 2012. Review of the genus Andropogon (Poaceae: Andropogoneae) in America based on cytogenetic studies. Journal of Botany 2012(632547): 1-9. DOI: https://doi.org/10.1155//2012/632547

Reichert, E. T. 1913. The differentiation and specificity of starches in relation to genera, species, etc.; stereochemistry applied to protoplasmic processes and products, and as a strictly scientific basis for the classification of plants and animals, No. 173, part I. The Carnegie Institution of Washington. Washington, D.C., USA. Pp. 342. DOI: https://doi. org/10.5962/bhl.title.24351

Sarwar, M. H., M. F. Sarwar, M. Sarwar, N. A. Qadri and S. Moghal. 2013. The importance of cereals (Poaceae: Gramineae) nutrition in human health: A review. Journal of Cereals and Oilseeds 4(3): 32-35. DOI: https://doi.org/10.5897/ JCO12.023

Soreng, R. J., P. M. Peterson, K. Romaschenko, G. Davidse, F. O. Zuloaga, E. Judziewicz, T. S. Filgueiras, J. I. Davis and O. Morrone. 2015. A worldwide phylogenetic classification of the Poaceae (Gramineae) Journal of Systematics and Evolution 53(2): 117-137. DOI: https://doi.org/10.1111/ jse. 12150

Tateoka, T. 1954. On the systematic significance of starch grains of seeds in Poaceae. Journal of Japanese Botany 29(11): 341347.
Tateoka, T. 1962. Starch grains of endosperm in Grass Systematics. Botanical Magazine. Tokyo 75(892): 377-383. DOI: https:// doi.org/10.15281/jplantres1887.75.377

Tetlow, I. J. and M. J. Emes. 2017. Starch biosynthesis in the developing endosperms of grasses and cereals. Agronomy 7(4): 1-43. DOI: https://doi.org/10.3390/agronomy7040081

Thiers, B. 2020. Index Herbariorum: a global directory of public herbaria and associated staff. New York Botanical Garden's Virtual Herbarium. Available at http://sweetgum.nybg.org/ ih/ (consulted January 2021).

Watson, L., T. D. Macfarlane and M. J. Dallwitz. 1992 (onwards). The grass genera of the world: descriptions, illustrations, identification, and information retrieval; including synonyms, morphology, anatomy, physiology, phytochemistry, cytology, classification, pathogens, world and local distribution, and references. https://www.deltaintkey.com/grass/refs.htm (consulted January 2021).

Welker, C. A. D., M. R. McKain, M. C. Estep, R. S. Pasquet, G. Chipabika, B. Pallangyo and E. A. Kellogg. 2020. Phylogenomics enables biogeographic analysis and a new subtribal classification of Andropogoneae (PoaceaePanicoideae). Journal of Systematics and Evolution 58(6): 1003-1030. DOI: https://doi.org/10.1111/jse.12691

Wipff, J. K. and R. B. Shaw. 2018. The taxonomic change in the Andropogon virginicus complex (Poaceae). Phytoneuron 2018-73: 1-2.

Yun, M. S. and Y. Kawagoe. 2010. Septum formation in amyloplasts produces compound granules in the rice endosperm and is regulated by plastid division proteins. Plant and Cell Physiology 51(9): 1469-1479. DOI: https://doi.org/10.1093/ pcp/pcq116 